University of Louisville

ThinkIR: The University of Louisville's Institutional Repository

\title{
Syndecan-1 tagged liposomes as a theranostic nanoparticle for pancreatic adenocarcinoma.
}

Wenyuan Yin

University of Louisville

Follow this and additional works at: https://ir.library.louisville.edu/honors

Part of the Biology Commons, and the Cancer Biology Commons

\section{Recommended Citation}

Yin, Wenyuan, "Syndecan-1 tagged liposomes as a theranostic nanoparticle for pancreatic adenocarcinoma." (2016). College of Arts \& Sciences Senior Honors Theses. Paper 126.

http://doi.org/10.18297/honors/126

This Senior Honors Thesis is brought to you for free and open access by the College of Arts \& Sciences at ThinkIR: The University of Louisville's Institutional Repository. It has been accepted for inclusion in College of Arts \& Sciences Senior Honors Theses by an authorized administrator of ThinkIR: The University of Louisville's Institutional Repository. This title appears here courtesy of the author, who has retained all other copyrights. For more information, please contact thinkir@louisville.edu. 
SYNDECAN-1 TAGGED LIPOSOMES AS A THERANOSTIC NANOPARTICLE

FOR PANCREATIC ADENOCARCINOMA

\author{
By Wenyuan Yin
}

Submitted in partial fulfillment of the requirements for Graduation (summa or magna) cum laude and

For Graduation with Honors from the Department of Biology

University of Louisville

May 2016 


\section{Acknowledgments}

I would like to thank my mentor, Dr. Lacey McNally, for several years of valuable instruction and guidance. I am immensely grateful for the opportunities she provided me as an undergraduate researcher, and for her support as I continue my scientific career. The skills and experiences I gained in her lab have prepared me well.

I would also like to thank my coworkers, Shanice Hudson, Charles Kimbrough, Anil

Khanal, and Bigya Khanal, for their assistance on my project. Furthermore, I thank Dr. William Grizzle at the University of Alabama, Birmingham, for his advice and assistance.

Finally, I would like to acknowledge the Rounsavall Foundation for funding this work. 


\begin{abstract}
Theranostic nanoparticles are emerging as a novel mechanism for detecting and treating cancer. Due to the difficulties in detection and treatment of pancreatic cancer, these particles could serve within this unique niche. In this study, a Syndecan-1 ligand was utilized to increase tumor specificity of fluorescent dye encapsulated liposomes which were evaluated as a potential theranostic nanoparticle for pancreatic adenocarcinoma. Their diagnostic capabilities and specificity to pancreatic adenocarcinoma were determined in vitro using immunocytochemistry and in vivo using multi-spectral optoacoustic tomography (MSOT). Immunocytochemistry showed that liposomes preferentially bound and released their contents into cells expressing high levels of Insulin-Like Growth Factor 1 Receptor. In an orthotopic pancreatic cancer mouse model, the liposomes preferentially targeted the pancreatic tumor with little off-target binding in the liver and spleen. Peak accumulation of the liposomes in the tumor occurred at $8 \mathrm{~h}$ post-injection. MSOT imaging was able to provide high-resolution 3D images of the tumor and liposome location. Ex vivo analysis showed that non-targeted liposomes accumulated in the liver suggesting that specificity of the liposomes for pancreatic adenocarcinoma was due to the presence of the Syndecan-1 ligand. Syndecan-1 tagged liposomes specifically target pancreatic adenocarcinoma both in vitro and in vivo. Once bound, the liposomes released the dye in vitro as indicated by red fluorescence of DNA-bound propidium iodide. The therapeutic drug-delivering capabilities of Syndecan-1 liposomes remain to be tested.
\end{abstract}




\section{Introduction}

Pancreatic ductal adenocarcinoma is a highly aggressive and almost universally fatal cancer with an average five-year survival rate of $5 \%$ across all stages (1). The poor prognosis of pancreatic cancer patients is due to inadequate early detection and ineffective treatments once diagnosed. Due to its asymptomatic nature in the early stages, over $85 \%$ of patients are diagnosed at stages III-IV with metastases, eliminating surgery as a curative option (1-2). Furthermore, ultrasound-guided needle biopsy, commonly used to diagnose pancreatic adenocarcinoma, requires suspected tumors to be at least $1 \mathrm{~cm}^{3}$ (3). The current gold-standard treatment for pancreatic cancer, gemcitabine, only enhances survival by 6.7 months. Advancements in chemotherapy such as FOLFIRONIX and nab-Paclitaxel minimally increase median survival, with survival rates of 11 and 8.5 months, respectively (4).

The underlying biology of pancreatic ductal adenocarcinoma can make these tumors particularly resistant to chemotherapy. The dense desmoplastic stroma and poor vascularity of these tumors inhibits the accumulation of traditional chemotherapies and prevents sufficient drug concentrations from being attained within the tumor (7). Nanoparticle drug carriers can be an effective strategy for overcoming these extracellular barriers to drug delivery by increasing the therapeutic load at tumor sites while decreasing systemic toxicity (8-10). Enhanced accumulation at pathological sites can result in an increase in the drug's therapeutic efficacy and reduction of incidence and intensity of side effects, resulting in improved patient acceptance, compliance and prognosis $(8,12-14)$.

Liposomes in particular have multiple desirable characteristics, such as the capacity for encapsulating large amounts of materials, the ability to protect these materials from degradation, and the capability for intracellular delivery through fusion with the plasma membrane (9). 
Liposomes are also permeable in environments with high collagen content, a trait of the extracellular matrices of solid pancreatic tumors (10-11). In addition to being drug carriers, liposomes can also encapsulate contrast agents for diagnostic imaging, thus combining diagnostic and therapeutic functions into one system. These particles, termed "theranostic nanoparticles", are a novel approach for detecting, monitoring, and treating cancer (12-15). The contrast agents loaded into theranostic nanoparticles allow tracking of nanoparticle accumulation and biodistribution using standard techniques such as planar fluorescent imaging (16). An important characteristic of theranostic nanoparticles is that a targeting ligand can be covalently attached to the outside of the particle so that it will preferentially accumulate at the tumor site (15-18).

Syndecan-1 (Sdc1) can be used to specifically target pancreatic adenocarcinoma due to its known interactions with Insulin-like Growth Factor 1 receptor (IGF1R) (19-21). IGF1R has been shown to be highly expressed in pancreatic adenocarcinoma and other metastatic cancers with an important role in cell proliferation, tumor metastasis, and anti-apoptotic pathways (22-26). High IGF1R expression correlates to resistance to chemotherapy and radiation-induced apoptosis and is a marker for poor prognosis in pancreatic, breast, lung, and prostate cancers (23-26). The ectodomain of Sdc1 binds to IGF1R to activate $\alpha_{v} \beta_{3}$ integrin in many aggressive cancers, a mechanism not found in non-malignant epithelial cells (20-21). Furthermore, Sdc1-tagged optical probes have already been shown to bind specifically to pancreatic tumor cells in vivo (27). Thus, covalently attaching $\mathrm{Sdc} 1$ to the outside of a liposome may produce a theranostic nanoparticle that is specific to pancreatic adenocarcinoma, potentially leading to an improved method of transporting imaging agents and chemotherapies directly to the tumor site.

Because Sdc1-tagged liposomes can encapsulate a variety of optically active molecules, or chromophores, they can be imaged using traditional imaging techniques (16). However, multi- 
spectral optoacoustic tomography (MSOT) offers several advantages over traditional nuclear imaging modalities. MSOT operates via the photoacoustic effect, wherein a medium excited by pulsed laser light subsequently emits an acoustic wave. Chromophores absorb a photon and enter an excited state, generating heat upon relaxation. This pulse of heat leads to an increase in temperature and local pressure, termed thermoelastic expansion. Propagation of the pressure differential results in the formation of an acoustic wave, which can then be recorded with ultrasound detectors to create high-resolution 3D images (28-29). Expansion of endogenous and exogenous chromophores occur at specific excitation wavelengths, allowing for selective imaging of compounds of interest. Due to the low-scattering characteristic of ultrasonic waves, images do not suffer from the light scattering or signal attenuation that limits optical and fluorescent imaging. Additional advantages include an enhanced spatial resolution of up to $150 \mu \mathrm{m}$ and accurate 3D imaging of deep tissue layers (29-33). Furthermore, multi-wavelength measurements can be used to quantify spatially varying concentrations of chromophores within biological tissues (28). MSOT can therefore be a valuable tool in determining the precise location and concentration of theranostic nanoparticles in vivo.

In this study, Syndecan-1 tagged liposomes were synthesized to encapsulate a near-infrared (NIR) fluorescent dye, CF-750 or propidium iodide (PI), to allow for tracking of the liposome in vivo or determine the potential of the liposome to release its cargo within a tumor cell, respectively (Fig. 1). As a further control within experiments of the Syndecan-1 tagged liposome, untargeted liposomes were utilized. Untagged liposomes had no targeting ligand.

The specificity of Sdc1 liposomes for pancreatic adenocarcinoma was evaluated both in vitro and in vivo. Immunocytochemistry was performed to visualize IGF1R-dependent binding and endocytosis of Sdc1 and untagged liposomes. Sdc1 liposomes were systemically injected by 
intravenous injection into an orthotopic mouse model to allow for in vivo tracking of biodistribution and accumulation. This study examines the feasibility of using Sdc1-tagged liposomes as a theranostic nanoparticle for pancreatic adenocarcinoma. 


\section{Materials \& Methods}

\section{Cell Culture}

Pancreatic adenocarcinoma cell lines S2VP10 and S2013, metastatic subclones of the SUIT-2 line, were obtained from Dr. Michael A. Hollingsworth at the University of Nebraska. Squamous cell carcinoma cell line SCC-1 was obtained from the University of Michigan. Pancreatic adenocarcinoma cell line MiaPaCa-2 was obtained from ATCC (Manassas, VA). S2VP10, S2013, and SCC-1 cells were cultured in RPMI 1640 and MiaPaCa-2 cells in DMEM medium (Gibco, Grand Island, NY), supplemented with 10\% Fetal Bovine Serum (Atlanta Biologicals, Flowery Branch, GA) and $1 \%$ L-Glutamine (Gibco). The cells were cultured at $37^{\circ} \mathrm{C}$ and $5 \% \mathrm{CO}_{2} . \mathrm{S} 2 \mathrm{VP} 10$ cells were infected with a retrovirus containing the firefly luciferase (Luc) gene (Stratagene, La Jolla, CA) and a luciferase-positive single cell clone, S2VP10L, was isolated (34).

\section{Synthesis of Liposomes}

\section{Materials}

Soybean L- $\alpha$-phosphatidylcholine $\quad$ (95\%) (PC), 1,2-dioleoyl-sn-glycero-3phosphoethanolamine (DOPE) and 1,2-dioleoyl-sn-glycero-3-phosphoethanolamine-N(hexanoylamine) (CAP) were purchased from Avanti Polar Lipids (Alabaster, AL). NHydroxysulfosuccinimide sodium salt (NHS), N-(3-Dimethylaminopropyl)-N'-ethylcarbodiimide hydrochloride $(E D C)$ and propidium iodide were purchased from Sigma (St. Louis, MO). CF-750 N-hydroxysuccinimide ester amine-reactive dye was purchased from Biotium (Hayward, CA). Finally, Syndecan-1 (Sdc1) was purchased from Prospec (Rehovot, Israel). 


\section{Synthesis of propidium iodide and CF-750 encapsulated liposomes}

Naked liposomes were synthesized by thin film hydration method (35). Soybean PC $(0.0067 \mathrm{~g})$, CAP $(0.0023 \mathrm{~g})$ and DOPE $(0.0020 \mathrm{~g})$ were dissolved in chloroform. Then, the chloroform was evaporated by heating at $70^{\circ} \mathrm{C}$ for $10 \mathrm{~min}$ in a rotary evaporator to get a thin film of lipids. The thin film was hydrated by adding $1 \mathrm{ml} \mathrm{PBS}(\mathrm{pH} 7.4,0.9 \% \mathrm{NaCl})$ and propidium iodide or CF-750 $(1 \mathrm{mM}, 0.1 \mathrm{ml})$ for $2 \mathrm{~h}$ at $70^{\circ} \mathrm{C}$. Then, the liposomes were sonicated for $2 \mathrm{~h}$ at $70^{\circ} \mathrm{C}$. Finally, liposomes were extruded four times through a polycarbonate filter $(200 \mathrm{~nm})$ at $70^{\circ} \mathrm{C}$.

\section{Conjugation of Syndecan-1 to dye-encapsulated liposomes}

Syndecan-1 was conjugated to the dye-encapsulated naked liposomes by carbodiimide chemistry. Syndecan-1 $(0.74 \mathrm{mM}, 20 \mu \mathrm{l})$ was mixed with NHS $(1 \mathrm{mM}, 0.1 \mathrm{ml})$ and EDC $(1 \mathrm{mM}$, $0.1 \mathrm{ml}$ ) and incubated for $6 \mathrm{~h}$ at room temperature at $\mathrm{pH} 7.5$. Then, $1 \mathrm{ml}$ of liposomes was added to this solution and mixed for 1 day at room temperature. The excess NHS and EDC were removed by dialysis, minimum 2000-3000 Da. The stock solution of Sdc1 conjugated liposomes was diluted further to get the desired concentration.

\section{Characterization of Liposomes}

\section{DLS measurements}

The size distribution of the liposomes was analyzed by dynamic light scattering (DLS) using a Zetasizer Nano-ZS (Malvern Instruments Ltd., Malvern, United Kingdom). The detection angle was $173^{\circ}$ and the incident beam was a $\mathrm{He}-\mathrm{Ne}$ ion laser $(\lambda=633 \mathrm{~nm})$. Correlation functions were analyzed by a histogram method and used to determine the diffusion coefficient (D) of the liposomes in the sample. Hydrodynamic radius (Rh) was calculated from D by using StokesEinstein's equation: 


$$
R_{h}=\frac{K_{b} T}{6 \pi \eta D}
$$

where $\mathrm{kB}$ is Boltzmann's constant, $\mathrm{T}$ is absolute temperature, and $\eta$ is solvent viscosity (36).

\section{Zeta-potential measurements}

Electrophoretic mobility $(\mu \mathrm{E})$ of the liposomes was measured at $25{ }^{\circ} \mathrm{C}$ with the Zetasizer Nano-ZS. The zeta-potential of the samples was calculated from $\mu \mathrm{E}$ using following Smoluchowski's equation:

$$
\mu \mathrm{E}=\frac{\zeta \varepsilon}{\eta}
$$

where $\zeta$ is the zeta-potential, $\varepsilon$ the permittivity of solvent, and $\eta$ the solvent viscosity (36).

\section{Transmission electron microscopy}

$10 \mu \mathrm{L}$ of $1 \mathrm{mM}$ liposomes were dropped on 200 mesh copper grids (Electron Microscopy Sciences, Hatfield, PA) and dried at room temperature for $5 \mathrm{~h}$. Images were collected using the Phillips CM-10 transmission electron microscope at $80 \mathrm{kV}$, equipped with a 15 megabyte SIA digital camera.

\section{UV-Vis spectroscopy}

The absorption spectrum for the CF-750 encapsulated liposomes was analyzed via UVVisible spectroscopy. Absorption at wavelengths every $10 \mathrm{~nm}$ between 650 and $860 \mathrm{~nm}$ was measured using the NanoDrop 2000 Spectrophotometer (Thermo Scientific, Waltham, MA) and accompanying software. Near-infrared (NIR) fluorescence signal was confirmed using Li-Cor Odyssey infrared scanner (Li-Cor, Lincoln, NE).

\section{Western Blot Analysis}


Whole cell lysates from S2VP10L, MiaPaCa-2, S2013, and SCC-1 cells were collected to determine IGF1R expression. The cells were plated at a density of $5 \times 10^{5}$ cells per well in a 6 -well plate $24 \mathrm{~h}$ prior to protein harvest. Cells were then lysed in a solution containing $1 \% \mathrm{NP}-40,1 \%$ protease inhibitor, and $1 \%$ phosphatase inhibitor (Thermo) in deionized water. The lysates were centrifuged at 13,000 x $g$ for 10 min. Total protein concentration was determined via Bradford assay (Bio-Rad, Hercules, CA).

Approximately $50 \mu \mathrm{g}$ of total protein was dissolved in deionized water, loading buffer, and reducing agent (Life Technologies, Carlsbad, CA). Proteins were separated using NuPage 4-12\% Bis-Tris gel and transferred onto a nitrocellulose membrane by iBlot (Life Technologies). The membrane was blocked in blocking buffer (Li-Cor) for $30 \mathrm{~min}$ and then incubated overnight at $4^{\circ} \mathrm{C}$ with IGF1R antibody (Abcam, Cambridge, England) at a concentration of $1 \mu \mathrm{g} / \mathrm{ml}$ and $\beta$ Actin antibody (Thermo) at a concentration of 1:3000. The membrane was then washed $3 \mathrm{X}$ with TBS (20 mM Tris- $\mathrm{HCl}, 150 \mathrm{mM} \mathrm{NaCl}$ in $\mathrm{diH}_{2} \mathrm{O}$ ) for 10 min each, incubated with donkey antimouse IRDye 680RD and donkey anti-rabbit IRDye 800CW secondary antibodies (Li-Cor) for 1 h, washed $3 \mathrm{X}$ with TBS for 10 min each, and scanned using Li-Cor Odyssey infrared scanner. Dosimetry was performed using Li-Cor software.

\section{Immunocytochemistry}

S2VP10L and SCC-1 cells were each seeded into 3 wells of a chamber slide at a density of $3 \times 10^{5}$ cells/well. Cells were grown overnight in RPMI with 10\% FBS and 1\% L-Glutamine at $37^{\circ} \mathrm{C}$ with $5 \% \mathrm{CO}_{2}$. The cells were then serum-starved for $3 \mathrm{~h}$ by switching to RPMI with $1 \% \mathrm{BSA}$

(Fisher). Immunocytochemistry buffers were prepared as follows: $\mathrm{PBS}^{++}(0.5 \mathrm{mM} \mathrm{CaCl}$ and $\mathrm{MgCl}_{2}$ in PBS), $\mathrm{PBS}^{++++}$(97.9 mL PBS++, $2 \mathrm{~mL} \mathrm{10 \%} \mathrm{BSA} \mathrm{+} 90.08 \mathrm{mg}$ dextrose), and citrate 


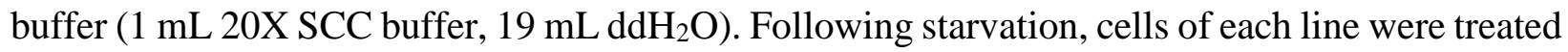
with $30 \mu \mathrm{L}$ PBS (control), $30 \mathrm{~L}$ of $1 \mathrm{mM}$ naked liposomes, or $30 \mu \mathrm{L}$ of $1 \mathrm{mM} \mathrm{Sdc} 1$ liposomes for $3 \mathrm{~h}$. Then, the wells were washed $2 \mathrm{X}$ with cold $\mathrm{PBS}^{++++}, 3 \mathrm{X}$ with ice cold citrate buffer, and again 2X with cold $\mathrm{PBS}^{++++}$. Cells were fixed with $4 \%$ paraformaldehyde (Electron Microscopy Sciences) for $5 \mathrm{~min}$ at $25^{\circ} \mathrm{C}$ followed by $15 \mathrm{~min}$ at $4^{\circ} \mathrm{C}$. Next, the cells were washed $6 \mathrm{X}$ with $\mathrm{PBS}^{++}$ for 10 min each. The slides were mounted in ProLong with DAPI (Invitrogen, Waltham, MA) and set overnight. Images were taken at 400X magnification with a Zeiss Photometer 2 (Carl Zeiss, Oberkochen, Germany) using DAPI, FITC, and Texas Red filters. The exposure times used were 20 ms DAPI, 200 ms FITC, and 200 ms Texas Red.

\section{Orthotopic Pancreatic Cancer Xenografts}

Strict adherence to the University of Louisville Institutional Animal Care and Use Committee (IACUC)-approved protocol was maintained throughout the study. Five-week old female C.B-17 SCID mice (Harlan Laboratories, Indianapolis, IN) were acclimated for one week prior to the study. Mice were anesthetized for all surgical procedures with $1.6 \%$ isoflurane and all procedures were performed in a sterile hood. Orthotopic cell implantation was performed as previously described $(30,34)$. A 1 -cm incision was made in the left upper abdominal quadrant and the spleen was located and used to indirectly position the tail of the pancreas, avoiding direct manipulation of the pancreas. The suspension of S2VP10L (Luc positive) cells in PBS was stored on ice and drawn up with a 28 -gauge needle. Five mice were each injected with $1.5 \times 10^{5} \mathrm{~S} 2 \mathrm{VP} 10 \mathrm{~L}$ (Luc positive) cells ( $30 \mathrm{uL}$ ) into the tail of the pancreas. Sterile cotton-tipped applicators were used to cover the injection site for $30 \mathrm{~s}$ to prevent peritoneal leakage. The organs were repositioned in the abdomen and the incision was closed using a single-layer closure with 5-0 nylon sutures. The 
mice recovered on a heated pad while receiving clear liquid acetaminophen for $24 \mathrm{~h}$ with food and water ad libitum. Confirmation of orthotopic implantation was performed using bioluminescence optical imaging on the Advanced Molecular Imager AMI-1000X (Spectral Imaging Instruments, Tucson, AZ). Mice with detectable leakage from the pancreas were removed from the study. Mice received intraperitoneal (IP) injection of $2.5 \mathrm{mg}$ luciferin $10 \mathrm{~min}$ prior to weekly imaging to monitor orthotopic tumor and metastatic growth. Region of interest (ROI) analysis was used to measure light emitted for orthotopic sites using the AMI Image Viewer software. Sutures were removed seven days following implantation. At 10 days post-implantation, mice were IV injected with $200 \mu \mathrm{L}$ of $100 \mathrm{nM}$ naked or Sdc1-tagged liposomes each containing CF-750 dye (Biotium).

\section{In Vivo Imaging and Reconstruction}

Multispectral optoacoustic tomographic (MSOT) imaging was performed as described by Kimbrough et al (27). Mice were anesthetized with $1.6 \%$ isoflurane and prepped for imaging with a combination of Nair cream with aloe (Church \& Dwight Co., Princeton, NJ) and shaving. The mice were imaged using MSOT system InVision TF 256 (iThera Medical, Munich, Germany). Serial slice images were taken in $0.2 \mathrm{~mm}$ steps between the diaphragm to the bottom of the kidneys $(40-55 \mathrm{~mm})$ at wavelengths of 680, 710, 730, 740, 760, 770, 780, 800, 850, and $900 \mathrm{~nm}$ using 25 averages per wavelength with acquisition time of $10 \mu \mathrm{sec}$ per frame in order to minimize the influence of animal movement. Images were taken every $8 \mathrm{~h}$ for $24 \mathrm{~h}$ to track liposome uptake and accumulation. Excitation of CF-750 liposomes was conducted using a tunable parametric oscillator pumped by an Nd:YAG laser. The pancreas tumor was identified by a live-feed screen preview multispectral signal. Video-rate acquisition produced an imaging clip compiled from single-slice acquisition in less than $1 \mathrm{~ms}$, resulting in an image data rate of 10 frames per second. Image 
reconstruction was conducted using backprojection at a resolution of $75 \mu \mathrm{m}$. Multispectral processing was conducted using linear regression (ViewMSOT 3.5). A 3.5mm diameter ellipse was used for region-of-interest analysis of liposome signal on the tumor, liver, and kidney.

\section{Ex Vivo Imaging of Organs}

Mice were euthanized $24 \mathrm{~h}$ after liposomal injections via carbon dioxide inhalation. The pancreas, liver, and spleen were harvested and imaged ex vivo to compare accumulation of naked and Sdc1 tagged liposomes. The organs were imaged using near-infrared (NIR) fluorescence with the AMI 1000X at $675 \mathrm{~nm}$ excitation and $760 \mathrm{~nm}$ emission. 


\section{Results}

\section{Western Blot}

Western blot analysis was performed to evaluate the expression of IGF1R on the three different pancreatic adenocarcinoma cell lines (S2VP10L, MiaPaCa-2, and S2013). The head and neck squamous cell carcinoma cell line SCC-1 served as a negative control (22). As expected, SCC-1 cells expressed much lower levels of IGF1R than pancreatic cancer lines (Fig. 2A). Less aggressive MiaPaCa-2 cells also had relatively low IGF1R expression. Highly aggressive and metastatic S2VP10L and S2013 cells had five- and seven-fold increase of IGF1R expression respectively compared to SCC-1 cells (Fig. 2B). S2VP10L cells were chosen as the pancreatic cell line to be used for the remainder of the experiments due to their predictable behavior in vivo and relatively high levels of IGF1R expression.

\section{Characterization of Liposomes}

Liposomes were synthesized to encapsulate CF-750 dye or propidium iodide and tagged with Syndecan-1. Transmission electron microscopy (TEM) of the liposomes shows the average size to be $117 \mathrm{~nm}$ (Fig. 3C). The size of liposomes observed by TEM is smaller than DLS (129 nm, Fig. 3A), as TEM yields a number-averaged size whereas DLS does a Z-averaged size that takes into account hydration layers. Based on the low polydispersion index, the liposomes are uniform in size (Fig. 3A). The liposomes also have a slight positive charge of $0.3 \mathrm{mV}$ (Fig. 3B).

\section{$U$ V-Vis Spectroscopy}

The absorption spectrum for CF-750 encapsulated Sdc1 liposomes was analyzed using UVVis spectroscopy. The peak absorption of the liposomes was $750 \mathrm{~nm}$, the same as the original dye (Fig. 4). Thus, encapsulating the dye within liposomes does not change the optical behavior of the dye. 


\section{Immunocytochemistry}

Immunocytochemistry slides of S2VP10L and SCC-1 cells were treated with non-targeted and Sdc1 liposomes containing propidium iodide and imaged using Texas Red, DAPI, and FITC filters. Fluorescence seen using the Texas Red filter indicates propidium iodide (PI) uptake by the cell. In IGF1R-positive S2VP10L cells, there was greatly increased uptake of PI when treated with Sdc1-tagged liposomes (Fig. 5C) compared to naked liposomes (Fig. 5B). Treatment with non-targeted liposomes does not result in the intracellular localization of dye, as the green fluorescence indicates extracellular accumulation (Fig. 5E) rather than intracellular uptake. The bright yellow areas in Figure 5F indicate locations where red and green light are both present, suggesting PI on both the cell surface as well as within the cytoplasm, where it is likely bound to mitochondrial DNA or free-floating RNA. White areas indicate red, green, and blue co-localization demonstrating the PI binding to nuclear DNA. Untreated S2VP10L cells show minimal red autofluorescence (Fig. 5A) and no green autofluorescence (Fig. 5D).

Propidium iodide, when bound to nucleic acids, displays maximum excitation and emission values at $538 \mathrm{~nm}$ and $617 \mathrm{~nm}$ respectively and fluoresces red using the Texas Red filter. However, PI in its free form exhibits different optical characteristics: maximum excitation at $488 \mathrm{~nm}$ and emission at $590 \mathrm{~nm}$ (37), fluorescing green using the FITC filter. This allows for distinction between excess dye bound on the cell surface versus internalized dye bound to nuclear or cytoplasmic nucleic acids.

\section{In Vivo Imaging and Reconstruction}

Syndecan-1-tagged liposomes were injected ten days post-implantation of the tumor. Liposome accumulation in vivo was determined using MSOT imaging every $8 \mathrm{~h}$ for $24 \mathrm{~h}$. 
Representative location of organs as seen on the MSOT can be viewed in Figure 6. Liposome accumulation in the tumor peaked at $8 \mathrm{~h}$ post-injection and declined within $24 \mathrm{~h}$ (Fig. 7C). The highest region of photoacoustic signal was between 45 and $46 \mathrm{~mm}$ in the mouse, and corresponded with the location of the pancreatic tumors (Fig. 7A). Low signal intensity was observed in the spleen and liver (Fig. 7B), indicating significantly more liposomes accumulated in the tumor versus in the liver and kidney $(\mathrm{p}<0.05)$. Furthermore, orthogonal views of the tumor demonstrate

probe accumulation along 3 spatial dimensions, and suggest liposome penetration and accumulation within the interior of the pancreatic tumor (Fig. 7B).

\section{Ex Vivo Imaging of Organs}

Ex vivo fluorescent imaging confirms accumulation of Sdc1-liposomes within organs (Fig. 8). Mice were euthanized $24 \mathrm{~h}$ post-liposomal injection. The pancreas, liver, and spleen were imaged using NIR fluorescent imaging with excitation $675 \mathrm{~nm}$ and emission $760 \mathrm{~nm}$. Sdc1-tagged liposomes bound preferentially to the pancreas tumor with little off-target binding in the liver and spleen. Non-targeted liposomes accumulated primarily within the liver. 


\section{Discussion}

This study investigated the feasibility of using Sdc1-tagged liposomes as a theranostic nanoparticle for detection and possible treatment of pancreatic adenocarcinoma. By utilizing fluorescent dyes, liposome specificity and unloading capability was assessed in vitro using immunocytochemistry. Accumulation time and specificity in vivo were analyzed by injecting the liposomes into an orthotopic mouse model and imaging using MSOT and ex vivo fluorescence imaging.

Treatment with liposome-encapsulated therapeutics has been shown to increase bioavailability of therapeutic agents at the tumor site, reduce off-target toxicity, and increase circulation time (39-41). Many of these liposomes utilize the Enhanced Permeability and Retention effect (EPR), a phenomenon wherein particles around $100 \mathrm{~nm}$ accumulate in tumors due to leaky vasculature, to passively target cancerous tissues (38). Sdc1-tagged liposomes were $117 \mathrm{~nm}$ in diameter, theoretically allowing them to diffuse into tumor sites via EPR. However, targeting IGF1R with the Sdc1 ligand utilizes a second mechanism to enhance specificity, active targeting.

Previous studies have shown that attaching a targeting ligand increases tumor targeting and intracellular uptake in tumor sites when compared to non-targeted liposomes (42). In this project, Sdc1-tagged liposome and control (untagged) liposome specificities were compared using ex vivo analysis. Though some control liposomes may have reached the pancreatic tumor due to passive targeting from EPR and the leakiness of the blood vessels around the tumor, Sdc1-tagged liposomes showed greater accumulation within the pancreatic tumor and less off-target binding. These results, congruent with previous findings, suggest that the active targeting of the Sdc1 ligand contributes most to the specificity of the Sdc1-tagged liposomes. 
Cancer cells treated with targeted liposomes containing chemotherapeutic agents show enhanced cell death when compared to non-targeted liposomes due to enhanced receptor-mediated endocytosis (43). This project showed that Sdc1-tagged liposomes are capable of fusing with cell membranes and unloading their contents into the cell, indicated by the presence of DNA-bound propidium iodide in S2VP10L cells treated with the liposomes. This result was observed in IGF1Rpositive S2VP10L cells and not in IGF1R-negative SCC1 cells, suggesting that the Sdc1-IGF1R interaction facilitates endocytosis.

The specific unloading of liposomal contents to IGF1R-positive cells in vitro and low offtarget binding in vivo demonstrate the feasibility of using Syndecan-1 as a targeting ligand for a pancreatic theranostic nanoparticle. Because Sdc1-tagged liposomes specifically target and preferentially release contents to pancreatic adenocarcinoma, encapsulating a drug in these particles can reduce systemic toxicity, leading to decreased side effects and improved patient prognosis $(8,12-14)$. Furthermore, the presence of a contrast agent allows for tracking of liposomal movement and accumulation and can be used to image pancreatic cancer in earlier stages using existing ultrasonic methods.

In this study, photoacoustic imaging was used to produce high resolution molecular images in vivo based on the absorption of contrast agents. It has previously been used to image both endogenous (such as melanin in the monitoring of melanoma) and exogenous (NIR dyes) chromophores in the context of cancer (44). Though photoacoustic imaging is not currently widely used in the clinic, applications of MSOT in clinical settings have begun with the development of a handheld MSOT system (45). Using MSOT to image theranostic nanoparticles may soon become a versatile tool in the diagnosis and monitoring of pancreatic cancer by providing a method of detecting tumors at an earlier stage. 
In conclusion, Syndecan-1 tagged liposomes actively target pancreatic adenocarcinoma with minimal off-target binding in vivo and are capable of releasing their contents in vitro. Though this study reveals that Sdc1-tagged liposomes can be useful for imaging pancreatic adenocarcinoma in a laboratory setting, further work is needed to assess their clinical value. Such studies should include using clinically viable techniques to image the liposomes evaluating the therapeutic capabilities of these liposomes. Ultimately these studies may lead to the development of a theranostic nanoparticle for clinical use. 


\section{References}

1. Howlader N, Noone AM, Krapcho M, Garshell J, Neyman N, Altekruse SF, Kosary CL, Yu M, Ruhl J, Tatalovich Z, Cho H, Mariotto A, Lewis DR, Chen HS, Feuer EJ, Cronin KA (eds). SEER Cancer Statistics Review, 1975-2010, National Cancer Institute. Bethesda, MD, http://seer.cancer.gov/csr/1975_2010/, based on November 2012 SEER data submission, posted to the SEER web site, April 2013.

2. Li, D, Xie, K, Wolff, R, \& Abbruzzese, JL (2004). Pancreatic cancer. The Lancet 2004 Mar;363(9414):1049-57

3. Agarwal B, Abu-Hamda E, Molke KL, Correa AM, Ho L. Endoscopic ultrasound-guided fine needle aspiration and multidetector spiral $\mathrm{CT}$ in the diagnosis of pancreatic cancer. Am J Gastroenterol. 2004 May;99(5):844-50.

4. Burris HA 3rd, Moore MJ, Andersen J, Green MR, Rothenberg ML, Modiano MR, Cripps MC, Portenoy RK, Storniolo AM, Tarassoff P, Nelson R, Dorr FA, Stephens CD, Von Hoff DD. Improvements in survival and clinical benefit with gemcitabine as firstline therapy for patients with advanced pancreas cancer: a randomized trial. J Clin Oncol. 1997 Jun;15(6):2403-13

5. Arslan C, Yalcin S. Current and future systemic treatment options in metastatic pancreatic cancer. J Gastrointest Oncol. 2014 Aug;5(4):280-295

6. Mohammed S, Van Buren Ii G, Fisher WE. Pancreatic cancer: Advances in treatment. World J Gastroenterol. 2014 Jul;20(28):9354-9360.

7. Long J, Zhang Y, Yu X, Yang J, LeBrun DG, Chen C, Yao Q, Li M. Overcoming drug resistance in pancreatic cancer. Expert Opin Ther Targets, 2011 Jul;15(7):817-28 
8. Schleich N, Po C, Jacobs D, Ucakar B, Gallez B, Danhier F, Préat V. Comparison of active, passive and magnetic targeting to tumors of multifunctional paclitaxel/SPIOloaded nanoparticles for tumor imaging and therapy. J Control Release. 2014 Nov 28;194:82-91

9. Lee RJ, Low PS. Delivery of liposomes into cultured KB cells via folate receptormediated endocytosis. J Biol Chem. 1994 Feb 4;269(5):3198-204.

10. Yokoi K, Kojic M, Milosevic M, Tanei T, Ferrari M, Ziemys A. Capillary-wall collagen as a biophysical marker of nanotherapeutic permeability into the tumor microenvironment. Cancer Res, 2014 May; pii: canres.3494.2013

11. Hidenori M, Tatsuya M, Kunihiro T, Hiromu S, Hidenori M, Seiki T. Tumor-Stroma Interaction of Human Pancreatic Cancer: Acquired Resistance to Anticancer Drugs and Proliferation Regulation Is Dependent on Extracellular Matrix Proteins. Pancreas, 2004 Jan;28(1):38-44

12. Rizzo LY, Theek B, Storm G, Kiessling F, Lammers T. Recent progress in nanomedicine: therapeutic, diagnostic, and theranostic applications. Curr Opin Biotechnol. 2013 Dec;24(6):1159-66

13. Patra CR, Bhattacharya R, Wang E, Katarya A, Lau JS, Dutta S, Muders M, Wang S, Buhrow SA, Safgren SL, Yaszemski MJ, Reid JM, Ames MM, Mukherjeel P, Mukhopadhyay D. Targeted Delivery of Gemcitabine to Pancreatic Adenocarcinoma Using Cetuximab as a Targeting Agent. Cancer Res, 2008 March;68(6):1970-8

14. He Y, Zhang L, Zhu D, Song C. Design of multifunctional magnetic iron oxide nanoparticles/mitoxantrone-loaded liposomes for both magnetic resonance imaging and targeted cancer therapy. Int J Nanomedicine. 2014 Aug 22;9:4055-66 
15. Fang C, Zhang M. Nanoparticle-based theragnostics: Integrating diagnostic and therapeutic potentials in nanomedicine. J Control Release, 2010 Aug;146(1):2-5

16. Ryu JH, Koo H, Sun IC, Yuk SH, Choi K, Kim K, Kwon IC Fang C, Miqin Z. Tumortargeting multi-functional nanoparticles for theragnosis: new paradigm for cancer therapy. Adv Drug Deliv Rev, 2012 Oct;64(13):1447-58

17. Hwang DW, Ko HY, Lee JH, Kang H, Ryu SH, Song IC, Lee DS, Kim S. A NucleolinTargeted Multimodal Nanoparticle Imaging Probe for Tracking Cancer Cells Using an Aptamer. J Nucl Med, 2010 Jan;51(1):98-105

18. Zeiderman MR, Egger ME, Kimbrough CW, England CG, Dupre TV, McMasters KM, McNally LR. Targeting of BRAF resistant melanoma via extracellular matrix metalloproteinase inducer receptor. J Surg Res, 2014 Jul;190(1):111-8

19. Rapraeger AC. Synstatin: a selective inhibitor of the syndecan-1-coupled IGF1R- $\alpha$ v $\beta 3$ integrin complex in tumorigenesis and angiogenesis. FEBS J, May 2013;280(10): 220715

20. Beauvais DM, Burbach BJ, Rapraeger AC. The syndecan-1 ectodomain regulates alphavbeta3 integrin activity in human mammary carcinoma cells. J Cell Biol, 2004;167:171-181

21. Beauvais DM, Rapraeger AC. Syndecan-1 couples the insulin-like growth factor-1 receptor to inside-out integrin activation. J Cell Sci, 2010 Nov;123(Pt 21):3796-807

22. Ouban A, Muracab P, Yeatman T, Coppola D. Expression and distribution of insulin-like growth factor-1 receptor in human carcinomas. Human Pathology, 2003 Aug;34(8):803-8

23. Turner BC, Haffty BG, Narayanan L, Yuan J, Havre PA, Gumbs AA, Kaplan L, Burgaud JL, Carter D, Baserga R, Glazer PM. Insulin-like growth factor-I receptor overexpression 
mediates cellular radioresistance and local breast cancer recurrence after lumpectomy and radiation. Cancer Res, 1997 Aug;57(15):3079-83

24. Huang JS, Egger ME, Grizzle WE, McNally LR. MicroRNA-100 regulates IGF1receptor expression in metastatic pancreatic cancer cells. Biotech Histochem, 2013 Oct;88(7):397-402

25. Hirakawa T, Yashiro M, Murata A, Hirata K, Kimural K, Amano R, Yamadal N, Nakata B, Hirakawa K. IGF-1 receptor and IGF binding protein-3 might predict prognosis of patients with resectable pancreatic cancer. BMC Cancer, 2013 Aug;13:392

26. Valsecchi ME, McDonald M, Brody JR, Hyslop T, Freydin B, Yeo CJ, Solomides C, Peiper SC, Witkiewicz AK. Epidermal growth factor receptor and insulinlike growth factor 1 receptor expression predict poor survival in pancreatic ductal adenocarcinoma. Cancer, 2012 Jul;118(14):3484-93

27. Kimbrough CW, Hudson S, Khanal A, Egger ME, McNally LR. Orthotopic pancreatic tumors detected by optoacoustic tomography using Syndecan-1. J Surg Res. Oct 2014; doi: $10.1016 /$ j.jss.2014.06.045

28. Cox B, Laufer JG, Arridge SR, Beard PC. Quantitative spectroscopic photoacoustic imaging: a review. Biomed Opt. 2012 Jun;17(6):061202. doi: 10.1117/1.JBO.17.6.061202.

29. Razansky D, Deliolanis NC, Vinegoni C, Ntziachristos V. Deep tissue optical and optoacoustic molecular imaging technologies for pre-clinical research and drug discovery. Curr Pharm Biotechnol. 2012 Mar;13(4):504-22

30. Hudson SV, Huang JS, Yin W, Albeituni S, Rush J, Khanal A, Yan J, Ceresa BP, Frieboes HB, McNally LR. Targeted Noninvasive Imaging of EGFR-Expressing 
Orthotopic Pancreatic Cancer Using Multispectral Optoacoustic Tomography. Cancer Res. 2014 Nov 1;74(21):6271-9

31. Buehler A, Herzog E, Ale A, Smith BD, Ntziachristos V, Razansky D. High resolution tumor targeting in living mice by means of multispectral optoacoustic tomography. EJNMMI Res, 2012 Apr;2:14

32. Lutzweiler C, Razansky D. Optoacoustic imaging and tomography: reconstruction approaches and outstanding challenges in image performance and quantification. Sensors, 2013 Jun;13(6):7345-84

33. Ntziachristos V, Razansky D. Molecular imaging by means of multispectral optoacoustic tomography (MSOT). Chem Rev, 2010 May;110(5):2783-94

34. McNally LR, Welch DR, Beck BH, Stafford LJ, Long JW, Sellers JC. KISS1 overexpression suppresses metastasis of pancreatic adenocarcinoma in a xenograft mouse model. Clin Exp Metastasis 2010;27(8):591-600.

35. Dua JS, Rana AC, Bhandari AK. Liposome: Methods of Preparation and Applications. Int J Pharm Sci Res. 2012 Apr;3(2): 14-20

36. Khanal A, Yusa S, Nakashima K. Fabrication of nanoaggregates of a triple hydrophilic block copolymer by cetyltrimethylammonium chloride binding. Langmuir. 2007 Oct 9;23(21):10511-

37. Krishan A, Ganapathi RN, Israel M. Effect of adriamycin and analogs on the nuclear fluorescence of propidium iodide-stained cells. Cancer Res. 1978 Nov;38(11 Pt 1):365662 
38. Maeda H, Greish K, Fang J. 2006. The EPR effect and polymeric drugs: A paradigm shift for cancer chemotherapy in the 21st century. Satchi-Fainaro R, Duncan, R. Polymer Therapeutics II. vol. 193 BerlinHeidelberg: Springer, pp. 103-121

39. Kim DH, Moon C, Oh SS, Park S, Jeong JW, Kim S, Lee HG, Kwon HJ, Kim KD. Liposome-Encapsulated CpG Enhances Antitumor Activity Accompanying the Changing of Lymphocyte Populations in Tumor via Intratumoral Administration. Nucleic Acid Ther. 2015 Feb 18. doi: 10.1089/nat.2014.0509

40. Andey TA, Sudhakar G, Marepally SK, Patel AR, Banerjee R, Sachdeva MS. Lipid Nanocarriers of a Lipid-conjugated Estrogenic Derivative Inhibit Tumor Growth and Enhance Cisplatin Activity against Triple-Negative Breast Cancer: Pharmacokinetic and Efficacy evaluation. Mol Pharm. 2015 Feb 8. [Epub ahead of print]

41. Kroon J1, Buijs JT, van der Horst G, Cheung H, van der Mark M, van Bloois L, Rizzo LY, Lammers T, Pelger RC, Storm G, van der Pluijm G, Metselaar JM. Liposomal delivery of dexamethasone attenuates prostate cancer bone metastatic tumor growth In Vivo. Prostate. 2015 Feb 8. doi: 10.1002/pros.22963. [Epub ahead of print]

42. Watanabe K1, Kaneko M, Maitani Y. Functional coating of liposomes using a folatepolymer conjugate to target folate receptors. Int J Nanomedicine. 2012;7:3679-88

43. Mallidi S, Luke GP, Emelianov S. Photoacoustic imaging in cancer detection, diagnosis, and treatment guidance. Trends Biotechnol. 2011 May;29(5):213-21

44. Buehler A, Kacprowicz M, Taruttis A, Ntziachristos V. Real-time handheld multispectral optoacoustic imaging. Opt Lett. 2013 May 1;38(9):1404-6 


\section{Figures}



Figure 1. Structure of Syndecan-1 tagged liposome encapsulating either propidium iodide or CF750 dye. 



Figure 2. Western blot analysis of pancreatic cell lines S2VP10L, MiaPaCa-2, S2013, and head and neck squamous cell carcinoma cell line SCC-1. (A) Western blot bands of $\beta$-Actin (42 kDa) and IGF1R (90 kDa). (B) Western blot band intensity dosimetry. The signal intensity of the IGF1R band was divided by the signal intensity of the $\beta$-Actin band to calculate relative abundance. Relative abundance of IGF1R in negative control SCC-1 cells was approximately 0.06. Pancreatic cell lines S2VP10L and S2013 displayed relative abundance of IGF1R of 0.32 and 0.42 respectively, a five- and seven-fold increase compared to the negative control. 

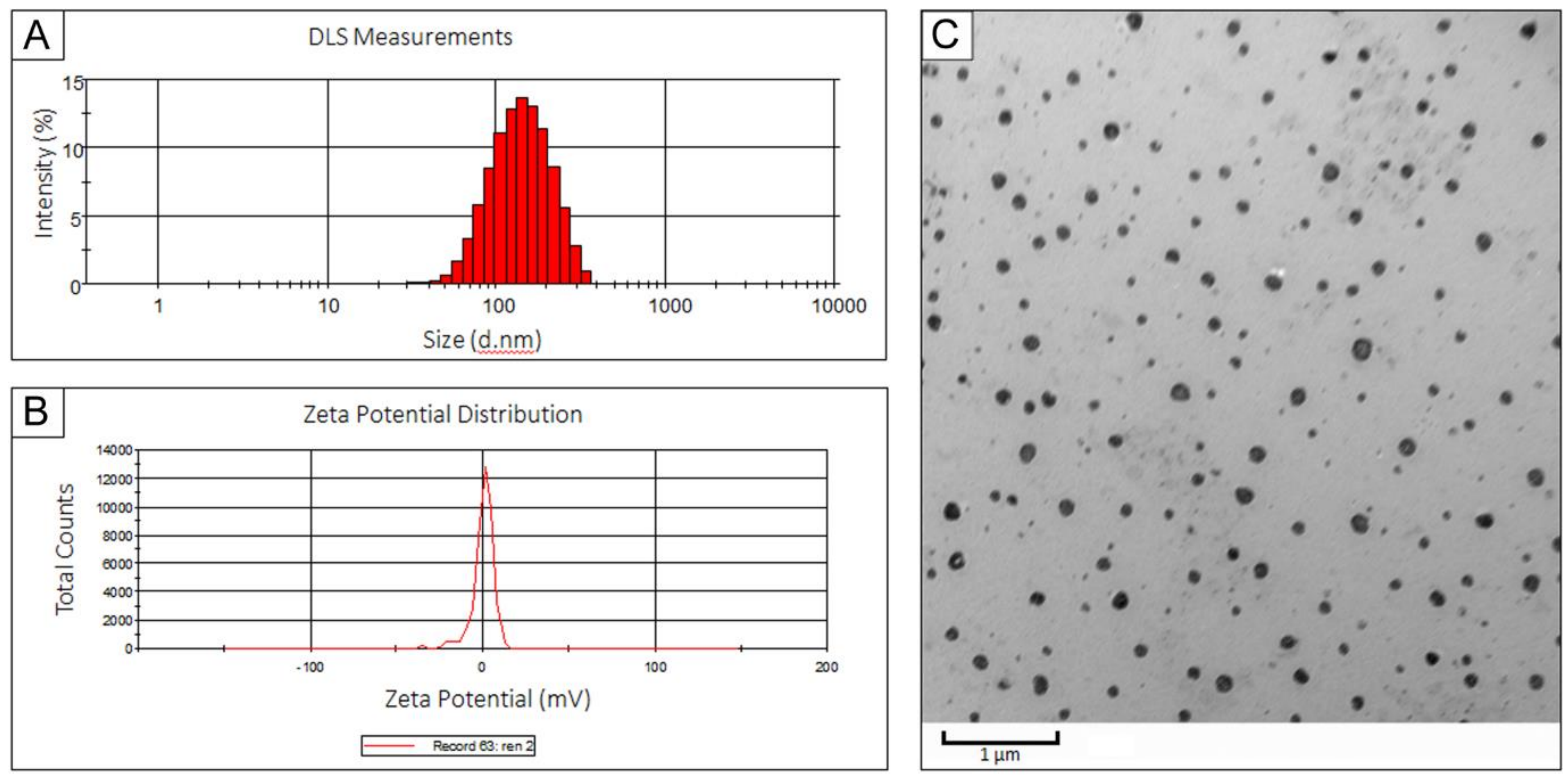

Figure 3. (A) DLS measurements to determine the size of the liposomes. The liposomes are approximately $129 \mathrm{~nm}$ in diameter with a polydispersion index of 0.05. (B) Zeta-potential measurements of the liposomes. $\mathrm{Z}=0.3 \mathrm{mV}$. (C) Transmission Electron Microscopy of the liposomes. The average size of the liposomes as determined by TEM is approximately $117 \mathrm{~nm}$. 




Figure 4. Absorption spectrum for CF-750 encapsulated Sdc1 liposomes. The liposomes demonstrated fluorescence activity with peak absorbance at $750 \mathrm{~nm}$. Encapsulating the CF-750 dye within the Sdc1 liposomes did not change the optical activity of the dye. 




Figure 5. S2VP10L cells following $3 \mathrm{~h}$ treatment at 400X magnification. All images were taken with the same exposure times. (A), (B), (C): Texas Red filter only. (D), (E), (F): Texas Red, DAPI, and FITC (A) Control cells show minimal red autofluorescence. (B) Cells treated with nontargeted liposomes display faint red signal, corresponding to minimal dye uptake. (C) Cells treated with Sdc1 liposomes show much stronger red fluorescence, indicating uptake of PI and binding of PI to DNA. (D) Control cells with DAPI. (E) Cells treated with non-targeted liposomes. Green signal is due to unbound PI, showing dye accumulation outside of the cell. (F) Cells treated with Sdc1 liposomes. Yellow signal occurs due to colocalization of red and green fluorescence, indicating that dye was located both on the cell surface and in the cytoplasm. White signal is colocalization of red, green, and DAPI signal. 


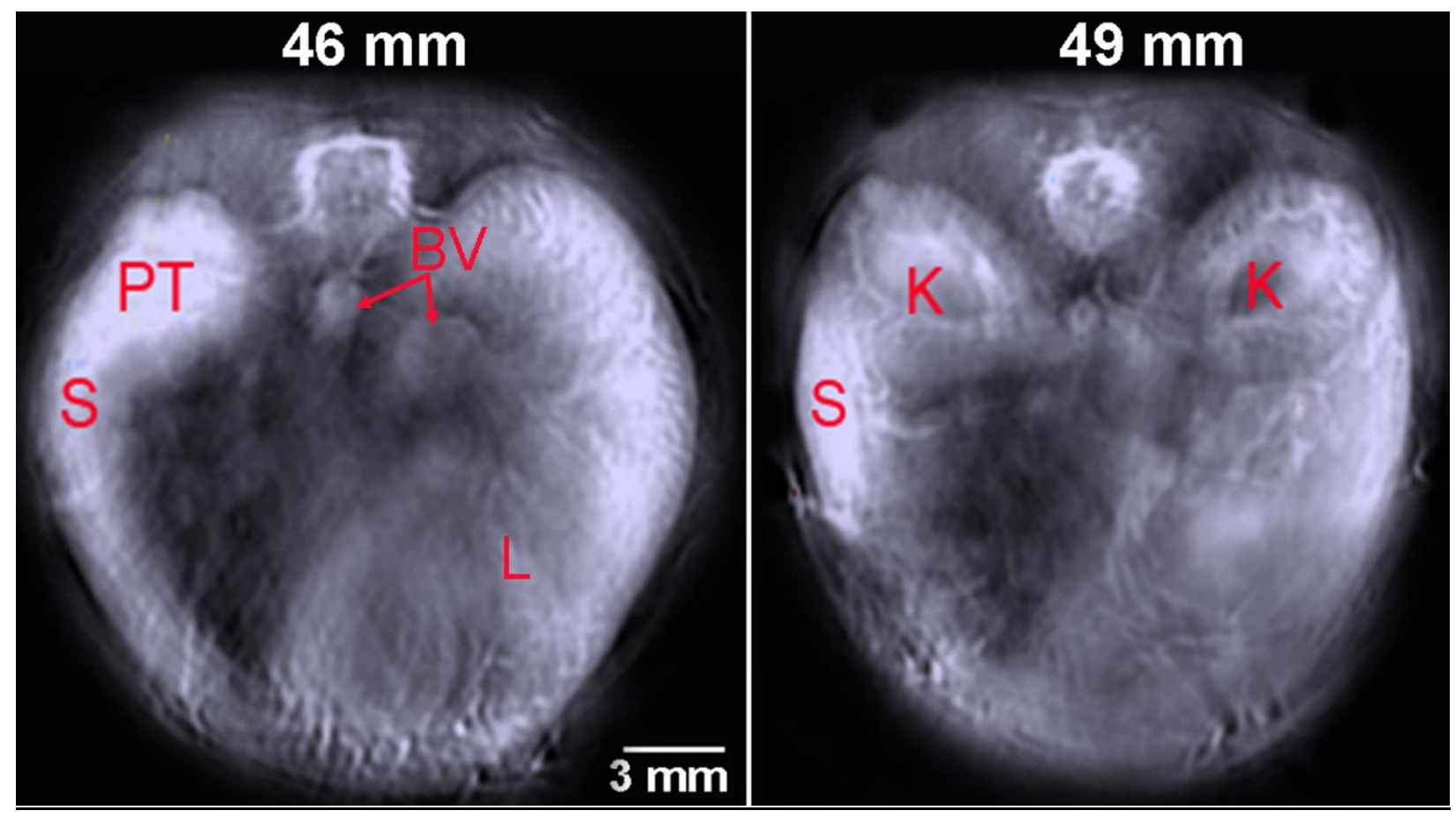

Figure 6. Representative locations of organs on MSOT at both 46 and $49 \mathrm{~mm}$. 

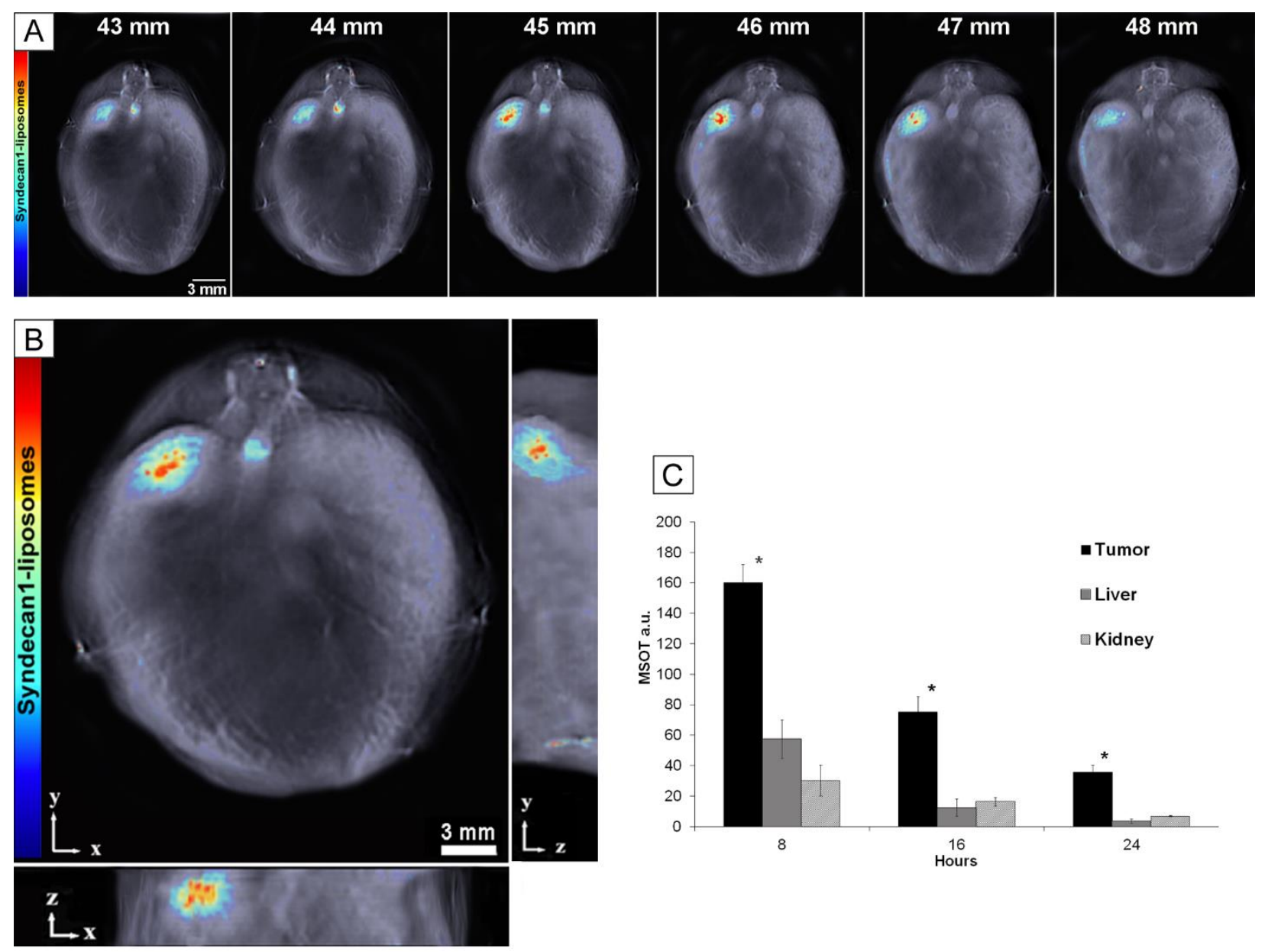

Figure 7. (A) Serial slice images of liposomal accumulation taken from 43 to $48 \mathrm{~mm}$ (abdomen). The highest signal intensity of the liposomes was at 45-46 $\mathrm{mm}$. (B) Orthogonal views of the pancreatic tumor and liposomal accumulation through different anatomical planes. (C) ROI analysis on liposome signal in various locations over time measured in MSOT a.u.. Bar height represents the median value and error bars represent the standard deviation throughout the organ. Peak liposomal accumulation occurred at $8 \mathrm{~h}$ post-injection. Significantly more liposomes accumulated in the tumor versus off-target organs $(\mathrm{p}<0.05)$. Representative locations of organs can be viewed in Supplemental Figure 2. 


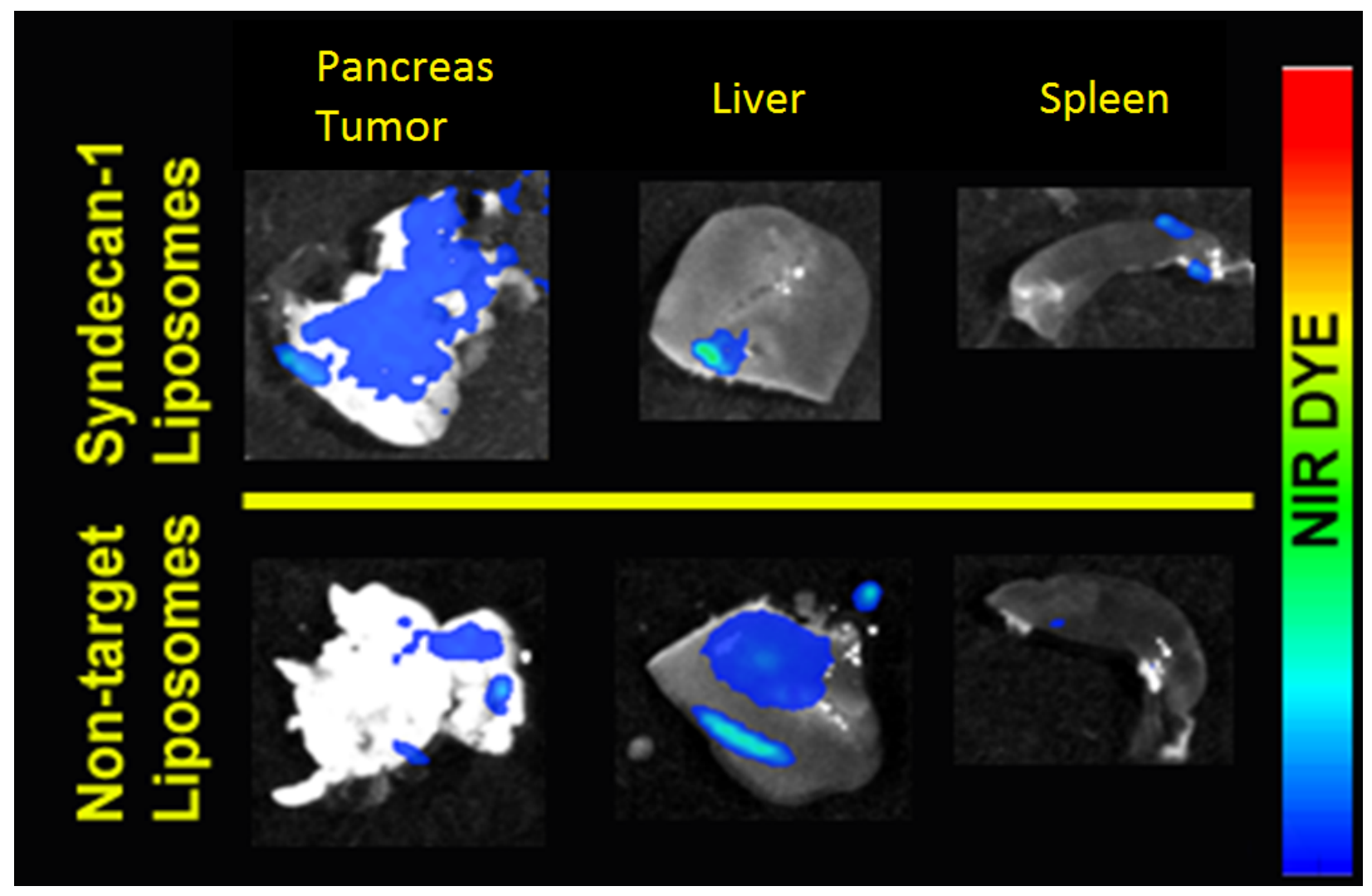

Figure 8. Ex vivo fluorescent imaging of the pancreas, liver, and spleen $24 \mathrm{~h}$ post-injection. Sdc1 liposomes accumulated in the tumor with very little off-target binding in the liver and spleen. Nontargeted liposomes tend to accumulate in the liver with low signal in the tumor. 